\title{
Cloud-Free Line-of-Sight Calculations
}

\author{
R. R. Rapp, C. SchUtz aNd E. Rodriguez \\ The Rand Corporation, Santa Monica, Calif. 90706
}

(Manuscript received 4 October 1972, in revised form 9 January 1973)

\section{ABSTRACT}

\begin{abstract}
A method for computing the probability of a cloud-free line-of-sight (CFLOS) between a given point in the atmosphere and a given point on the earth, was developed. The method is based on 3-hr synoptic weather reports of clouds, and whole-sky photographs taken during daylight hours over a period of three years at Columbia, Mo. The computational procedure is an effort to eliminate an apparent oversimplification in previously published methods that results from the use of mean cloud cover, an unsatisfactory vertical cloud distribution, and sunshine data. Present results are at variance with those earlier estimates, but compatible with recent observations actually taken from aircraft. Although the CFLOS estimates obtained are, by nature, uncertain, the range of uncertainty was estimated by using published empirical data and a quantitative error analysis.
\end{abstract}

\section{Introduction}

When there is an anticipated need to observe a point on the ground from high in the sky (or to observe the sky from a ground station), there may be a distinct advantage in foreknowing the probability of a cloudfree line-of-sight (CFLOS) for that geographical position. McCabe (1965) and Lund (1965) independently developed semi-objective methods for determining a

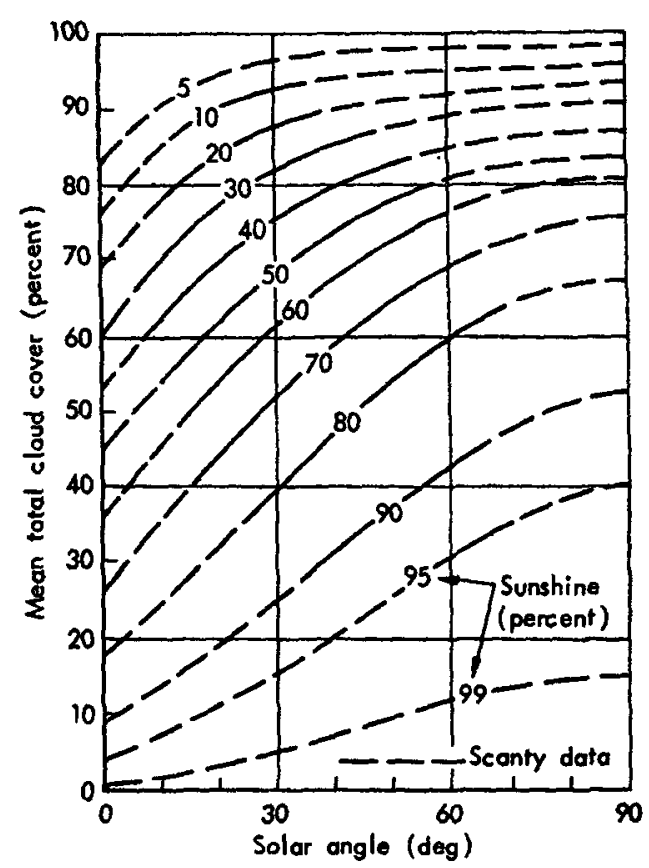

litc. 1. Approximate mean percentage of sunshine as a function of cloud cover and sun angle (after McCabe, 1965).
CFLOS probability, using only climatological data on clouds and sunshine. McCabe's results formed the basis for the calculation of CFLOS probabilities at various stations around the world (Quayle et al., 1968). However, a comparison of these data with data from actual aircraft observations (Bertoni, 1967) suggests that the Quayle estimates of probability were far too high.

McCabe's first assumption is that when bright sunshine is reported at the surface, there is a clear line of sight from the sun to the ground. Relating mean monthly cloud amounts at United States stations to mean number of hours of sunshine, and knowing solar elevation as a function of time, he constructed a graph (Fig. 1) from which could be read the probability of CFLOS as a function of look angle and cloud amount. Then assuming that the basic relationships among these three variables would apply for all cloud heights, McCabe devised a scheme for estimating the distribution of clouds as a function of height, based on the observations of DeBary and Möller (1963), which were made over central Europe.

An outstanding merit of McCabe's work, and also that of Lund (1966) as shown in Fig. 2, was that it recognized the important discrepancy between a ground observer's report of cloud amount and the probability of a CFLOS. Also, the concept of using sunshine data as a surrogate for CFLOS is reasonable, although there are discrepancies related to the observational technique that have been universally recognized.

The discrepancy between Quayle's results and Bertoni's observations derives from several effects. The presence of bright sunshine does not have a one-to-one relationship with the ability of a human eye to see an object on the ground. Actually, the CFLOS defined by 
McCabe neglects all consideration of "thin" clouds or surface haze and refers only to a path through the atmosphere that is free of opaque clouds; so CFLOS probabilities derived by McCabe's method should tend to be higher than clear line-of-sight (CLOS) probabilities as reported by Bertoni. Furthermore, studies by Appleman (1962) and Greaves et al. (1971) corroborate that cloudiness reported from above corresponds quite poorly with that reported from below.

When Quayle et al. used the McCabe results to estimate the CFLOS probabilities as many places in the world, they took mean monthly cloud amounts, entered McCabe's graph, and read out probabilities. But the relationship between cloud amounts and CFLOS is nonlinear for all but very shallow look angles, as is shown in Fig. 3 [derived from McCabe (1965)]; therefore, averaging the cloud amount before entering the graph can be shown to be poor procedure.

The difficulty is best demonstrated by a reductio ad absurdum. Suppose a location had 15 clear days and 15 overcast days in a month; in this case, both the mean cloudiness and the probability of a CFLOS would be 0.5 . If we enter Fig. 1, however, with 0.5 mean cloudiness and a look angle ("solar angle" in Fig. 1) of $90^{\circ}$ (either straight up or straight down), we arrive at a CFLOS probability of 0.9 .

We decided to perform a critical analysis of the McCabe method without changing the original hypothesis, which has gone a long way toward providing a realistic approach to the CFLOS problem. We have attempted to reduce some of the uncertainties that have arisen because of certain assumptions in the earlier CFLOS calculations. Specifically, this presentation does the following:

1) Treats a distribution of cloud cover instead of a mean cloud amount at the reporting station.

2) Considers the vertical distribution of the cloud cover using the best available data from an individual weather station.

3) Improves on the estimates made by $\mathrm{McCabe}_{\mathrm{C}}$ and others of relationships among look angle, cloud amount, and CFLOS probability at given ranges.

\section{Photogrammetric data}

Our methods were applied to data from Columbia, Mo., where CFLOS data were visually determined from whole-sky photographs compiled over a three-year period from 1 March 1966 to 28 February 1969 (Shanklin and Landwehr, 1971). ${ }^{1}$ The photographs were taken at 1-hr intervals to reveal the effect of diurnal variations on cloud cover and to permit correlation of these data with official Weather Bureau observations taken at the same location. They showed daytime cloud conditions at nine look angles, $10^{\circ}-90^{\circ}$, for the four

\footnotetext{
${ }^{1}$ The program was initiated and sponsored by the Air Force Cambridge Research Laboratories.
}



FIc. 2. Probability of a clear line-of-sight (sunshine) as a function of percent mean total cloud cover and look angle $\alpha$ when clear days are excluded from consideration (after Lund, 1966).

cardinal compass points starting with true north. The exact positions of the lines of sight on each print were readily located with a clear plastic overlay inscribed with 33 small circles whose centers represent 33 lines of sight at the given azimuths and look angles. Shanklin and Landwehr present graphs of CFLOS probability as a function of cloud cover in tenths and look angle for each azimuth and for all cloud types combined; the graphs indicate the realibility of the method. For example, the CFLOS probability at zero cloud cover, which actually represents cloud cover of less than $5 \%$, is greater than $96 \%$ at all look angles. Likewise, at $10 / 10$ clouds, or greater than $95 \%$ cloud cover, the probability is less than $9 \%$ at all look angles.

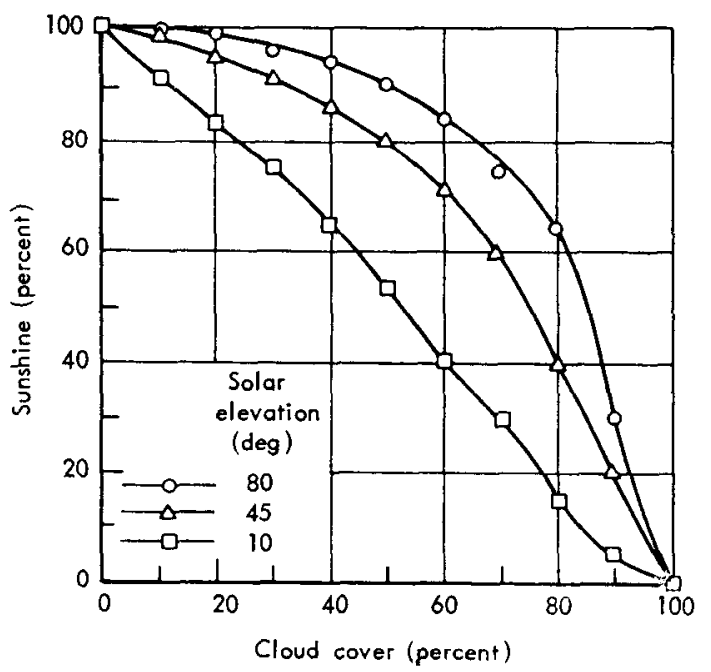

Fig, 3. Nonlinearity of relationship between CFLOS and cloud cover at three viewing angles (solar elevations). 
TABLE 1. Comparison of CFLOS probabilities from two sources.

\begin{tabular}{|c|c|c|c|c|c|c|c|c|c|}
\hline \multirow[b]{2}{*}{ Sources of data } & \multicolumn{9}{|c|}{ Cloud cover $k$} \\
\hline & $8 / 8$ & $7 / 8$ & $6 / 8$ & $5 / 8$ & $4 / 8$ & $3 / 8$ & $2 / 8$ & $1 / 8$ & $0 / 8$ \\
\hline & \multicolumn{9}{|c|}{ Elevation angle $30^{\circ}$} \\
\hline Shanklin/Landwehr & 0.06 & 0.27 & 0.43 & 0.56 & 0.68 & 0.76 & 0.85 & 0.92 & 0.99 \\
\hline McCabe & 0.00 & 0.20 & 0.40 & 0.58 & 0.72 & 0.82 & 0.90 & 0.96 & 1.00 \\
\hline \multirow[t]{2}{*}{ Difference } & +0.06 & +0.07 & +0.03 & -0.02 & -0.04 & -0.06 & -0.05 & -0.04 & -0.01 \\
\hline & \multicolumn{9}{|c|}{ Elevation angle $45^{\circ}$} \\
\hline Shanklin/Landwehr & 0.07 & 0.32 & 0.49 & 0.61 & 0.72 & 0.81 & 0.89 & 0.94 & 0.99 \\
\hline McCabe & 0.00 & 0.28 & 0.51 & 0.70 & 0.80 & 0.88 & 0.94 & 0.98 & 1.00 \\
\hline \multirow[t]{2}{*}{ Difference } & +0.07 & +0.04 & -0.02 & -0.09 & -0.08 & -0.07 & -0.05 & -0.04 & -0.01 \\
\hline & \multicolumn{9}{|c|}{ Elevation angle $60^{\circ}$} \\
\hline Shanklin/Landwehr & 0.08 & 0.35 & 0.52 & 0.67 & 0.76 & 0.83 & 0.90 & 0.95 & 0.99 \\
\hline McCabe & 0.00 & 0.33 & 0.62 & 0.77 & 0.85 & 0.93 & 0.96 & 0.99 & 1.00 \\
\hline Difference & +0.08 & +0.02 & -0.10 & -0.10 & -0.09 & -0.10 & -0.06 & -0.04 & -0.01 \\
\hline
\end{tabular}

Shanklin and Landwehr also computed the probabilities of CFLOS for each azimuth, look angle, and cloud type, on the basis of sunshine data recorded during the same three-year period by the Weather Bureau at Columbia, for each tenth of cloud cover. They found that, for all clouds, these sunshine-based probabilities of CFLOS varied with sunshine, increasing from an average of $12 \%$ at zero percent sunshine to $71 \%$ at $100 \%$ sunshine. These deviations, large when compared with the above-cited percentages derived from photographs, are due in part to a characteristic of the Weather Bureau's sunshine recorder: it does not detect thin clouds. Also, the recorder is directed solely toward the position of the sun in the sky (southerly azimuths).

For the present study, we averaged the CFLOS probabilities from the photographs for all azimuths and all clouds (Fig. 4) to permit direct comparison with McCabe's probabilities (Fig. 1). In Fig. 4 the curves were slightly smoothed. There were no data at less than $10^{\circ}$ elevation angle, so the character of the curve from $\sim 0^{\circ}$ to $10^{\circ}$ was assumed. Also, photogram data



Fig. 4. Probability (c) of CFLOS for all azimuths and all cloud covers (from Shanklin and Landwehr, 1971, Table 9). reached an unexplained maximum of CFLOS probability before $90^{\circ}$. This seems unrealistic and is probably due either to the way the data were observed, to the effects of lighting at the higher elevation angles, or to both. Therefore, we flattened the curves for higher elevation angles, beginning at the point of highest CFLOS. This point generally occurred at elevation angles $>60^{\circ}$. A more complete discussion of the data of Shanklin and Landwehr is given in Lund and Shanklin (1972).

We have used the CFLOS probability data in Fig. 4 for our calculations largely because these probabilities are based on carefully checked measurements of low, middle and high clouds. When these probabilities are averaged for all clouds and all azimuths, they fall generally within $10 \%$ of the McCabe estimates, as is shown in Table 1 . It should be noted that the averages from the photographs give a CFLOS probability up to $10 \%$ lower than that for sunshine data (Fig. 1) for cloud amounts $\leqslant 6 / 8(0.8)$ cloud cover, but up to $8 \%$ higher for greater cloud amounts, an important factor, particularly when considering cloudy areas.

\section{Synoptic data}

Data on the frequency of various amounts of cloud cover (in eighths ${ }^{2}$ ) for each cloud height considered in this paper (Table 2) were obtained from magnetic tapes in the Air Force TDF-14 format, prepared by the Environmental Technical Applications Center (ETAC). This format combines aviation reports with supplemental cloud data, every $3 \mathrm{hr}$. The data are available for all regularly reporting stations across the United States. (Overseas, the Air Force TDF-13 format is used.)

\footnotetext{
2 The choice of eighths of cloud as a breakdown was one of expedience, as many of the world's cloud data are reported in eighths. Lund (1965) achieved rather good results by merely separating clear days from days with cloud and using the mean cloud amount for the latter. It may therefore be reasonably assumed that a finer breakdown is unwarranted. A coarser breakdown, on the other hand, would necessitate additional manipulation of the cloud data before entering the calculation.
} 







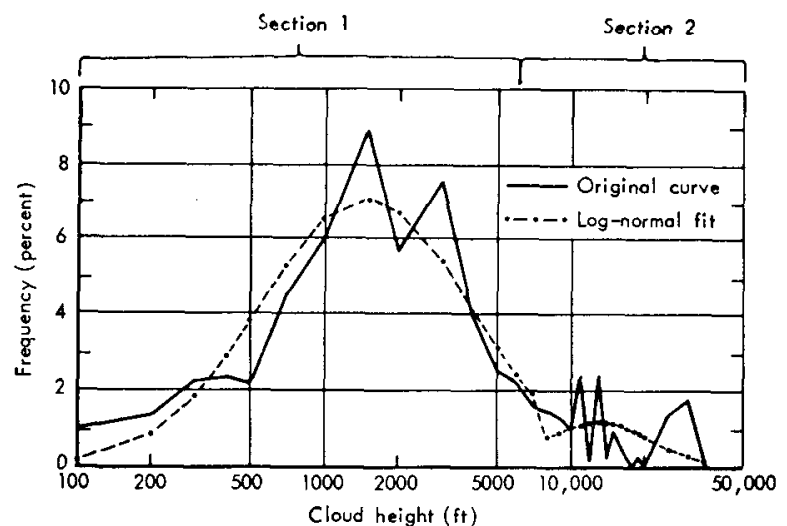

Fig. 5. Frequency of $8 / 8$ cloud cover at various altitudes over Columbia, Mo., (1945-68) in winter, and log-normal curves used for smoothing cloud-height data. See text for discussion of Sections 1 and 2 .

These tapes record synoptic data normally appearing on circuit "C." The program will accept records of any period greater than one year; however, the Columbia data covered a span from 1 January 1945 to 1 December 1968. The taped data were placed in the Rand Weather Data Bank (RAWDAB). They were then interrogated through a special set of computer programs developed by R. E. Huschke and E. Rodriguez for the cloud amount and height. The height listing eliminated the need for the DeBary and Möller (1963) vertical distribution of clouds.

\section{Method of calculation}

In order to reduce significantly the inaccuracies that result from using mean cloud amount values, because of the nonlinearity, we established the overall probability of a CFLOS for each reported cloud amount separately and then computed an average. To do this required 1) the RAWDAB frequency distribution of clouds to satisfy the function $f(l, k)$, where $l$ is the height of the cloud and $k$ the amount in eighths, and 2) the photographically-derived probability of a CFLOS as a function of look angle $\alpha$ and cloud amount $k$, represented by the function $c(\alpha, k)$. Now, if $f(l, k)$ represents the frequency of clouds of $k$ eighths at height $l$, and $c(\alpha, k)$ represents the probability of a CFLOS at a look angle $\alpha$ through clouds in the amount $k$, then the expected probability of a CFLOS from the surface to a given height (or from a given height to the surface) at a given look angle is the sum of the product of the frequency $f$ and the probability $c$, i.e.,

$$
P(\alpha, l)=\sum_{k=0}^{8} c(\alpha, k) f(l, k) .
$$

The next task was to examine critically the functions $c$ and $f$. In McCabe's original work, the value of $c(\alpha, k)$ was estimated from mean monthly cloud amounts and mean monthly sunshine records. Lund (1965) gave a thorough discussion of the entire problem, which details some of the subtleties of the CFLOS problem, and in a later paper (Lund, 1966), he presented a graph similar to McCabe's but based on the average cloudiness of only those days that were not completely clear. Lund's graph of the function labeled $c$ in this paper is shown in Fig. 2. As stated earlier, the aircraft observations reported by Bertoni (1967) were useful in calling into question the use of sunshine data, but since they were not related in a one-to-one fashion with ground observations, they could not be used effectively in constructing the function we desire. Shanklin and Landwehr (1971), however, present a wealth of data on the variations of CFLOS for all clouds as a function of look angle and cloud amount. Therefore, in our judgement, the function $c(\alpha, k)$ shown in Fig. 4 represents the best estimate available at this time.

If the function presented in Fig. 4 is the probability of seeing the ground from any altitude, as long as $k$ is interpreted as the cloud amount below the altitude in question, then the function $f(l, k)$ should represent the frequency that there are $k$ eighths of cloud below level $l$. The questionable validity of the DeBary and Möller distribution (used by McCabe) for areas other than central Europe has caused some concern over the use of the method. In order to eliminate this objection, the observed vertical and horizontal distributions of clouds were extracted from the same magnetic tapes of daily synoptic reports made available by ETAC. The difficulty that arises with this approach is that it is necessary to rely on the estimates of height made by ground observers. Estimates of cloud height and amount made by ground observers are deficient in three ways: low clouds obscure the extent of higher cloud coverage, the cloud-height reporting code does not provide a consistent scale, and observers tend to have biases in the heights that they do report. Historical weather records, therefore, do not give smooth distributions of cloud amount with height. The original $f(l, k)$ function for levels to $30,000 \mathrm{ft}$ exhibited clumping tendencies which were carried through to the computation of the CFLOS, thus generating irregular curves. Since there is no a priori reason to expect the true cloud distribution to be so discontinuous, we decided to apply a smoothing technique to the vertical distribution function. We found a log-normal distribution that nicely fits the low clouds and another log-normal that could fit the middle and high clouds. The final result is an equation, for each eighth of cloud cover, of the form

$$
f(l, k)=\left\{\begin{array}{llr}
a \ln N\left(m_{1}, \sigma_{1}\right), & \text { for } \quad 1 \leqslant l \leqslant 14 \\
b \ln N\left(m_{2}, \sigma_{2}\right), & \text { for } \quad 15 \leqslant l \leqslant 30
\end{array}\right.
$$

where $\ln N(m, \sigma)$ represents the normal distribution in the logarithm of height; the subscripts 1 and 2 refer to the lower $(<6,000 \mathrm{ft})$ and upper clouds $(6,000-30,000$ $\mathrm{ft}$, respectively (Table 2 ); and $a$ and $b$ are weighting factors depending on the relative amounts of lower and 
upper clouds. Fig. 5 shows the observed data for $8 / 8$ cloud cover over Columbia in the winter. The peaks reported for middle and high clouds are obvious, as is the discontinuous nature of the distribution of the low clouds. Also shown in Fig. 5 are the two sections of the component log-normal fit to the data. We believe that the smoothing achieved by the fitting procedure is probably a more realistic representation of cloud occurrence than is the obviously clumped data as reported. The mathematical details of the smoothing procedure for each $k$ tenths of cloudiness are as follows [where we define $x_{l}$ as the height of the upper bounds on interval $l, g_{l}$ as the frequency of cloud at height interval $l$, and $\left.\ln x_{l}=\log _{10} x_{l}\right]$ :

$g_{1 l}=\underset{6,000 \mathrm{ft}}{\text { normalized }} g_{l}$ with respect to intervals from 0 to

$$
=g_{l} / \sum_{l=1}^{14} g_{l}, \quad l=1,2, \ldots, 14
$$

$g_{2_{l}}=$ normalized $g_{l}$ with respect to intervals from 6,000 to $30,000 \mathrm{ft}$

$$
=g_{l} / \sum_{l=14}^{30} g_{l}, \quad l=14,15, \ldots, 30
$$

$\vec{x}_{1}=$ mean height for section 1

$$
=\sum_{l=1}^{14} g_{1_{l}} \ln x_{l}
$$

$\bar{x}_{2}=$ mean height for section 2

$$
=\sum_{l=14}^{30} g_{2 l} \ln x_{l}
$$

$\sigma_{1}=$ standard deviation for section 1

$$
=\left[\sum_{l=1}^{14} g_{1_{l}}\left(\ln x_{l}-\bar{x}_{1}\right)^{2}\right]^{\frac{1}{3}}
$$

$\sigma_{2}=$ standard deviation for section 2

$$
=\left[\sum_{l=14}^{30} g_{2 l}\left(\ln x_{l}-\bar{x}_{2}\right)^{2}\right]^{\frac{1}{3}} \text {. }
$$

Note that if all of the data reported in section $i$ ( 1 or 2 ) fall within one interval of height, then $\sigma_{i}=0$, and the approximation breaks down. Therefore, we followed the convention that $\sigma_{i}=0.25$ in such cases.

Let

$$
h(l, k)=\left\{\begin{array}{l}
\frac{1}{\sigma_{1} \sqrt{2 \pi}} \exp \left[-\left(\ln x_{l}-\overline{\ln x_{1}}\right)^{2} /\left(2 \sigma_{1}^{2}\right)\right], \\
\text { for } 1 \leqslant l \leqslant 14 \\
\frac{1}{\sigma_{2} \sqrt{2 \pi}} \exp \left[-\left(\ln x_{l}-\overline{\ln x_{2}}\right)^{2} /\left(2 \sigma_{2}^{2}\right)\right], \\
\text { for } 14 \leqslant l \leqslant 30 .
\end{array}\right.
$$

Thus, the approximation to the curve (0 to $35,000 \mathrm{ft}$ ) is the following combination of log-normal distributions:

$$
f^{\prime}(l, k)=\left\{\begin{array}{llr}
a h(l, k), & \text { for } \quad 1 \leqslant l \leqslant 14 \\
b h(l, k), & \text { for } \quad 15 \leqslant l \leqslant 30
\end{array}\right.
$$

where $a$ and $b$ are normalizing factors for sections 1 and 2 , respectively, and where

$$
\begin{aligned}
& a=\sum_{l=1}^{14} g_{l} / \sum_{l=1}^{30} g_{l}+g_{14}, \\
& b=\sum_{l=14}^{30} g_{l} / \sum_{l=1}^{30} g_{l}+g_{14 .}
\end{aligned}
$$

The approxination and the original curve are compared in Fig. 5.

The distribution function $f(l, k)$ was smoothed by using the above method for each fraction (in eighths) of cloud cover $(1 / 8$ to $8 / 8)$. A cumulative $D(l, k)$ table (Table 2) was then generated from the smoothed $f(l, k)$ table. Table 2 represents the probability of finding $k$ eighths of cloud cover at and below the height level $l$. The $0 / 8$ entry was computed as

$$
1-\sum_{k=1}^{8} D(l, k), \quad \text { for } \quad l=1,2, \ldots 30 .
$$

The entries in the cumulative table are used to compute the CFLOS at a given look angle $\alpha$, and below height level $l$ (Table 2 , three right-hand columns) :

$$
\operatorname{CFLOS}(\alpha, l)=\sum_{k=0}^{8} c(\alpha, k) D(l, k),
$$

where $c(\alpha, k)$ are CFLOS probabilities based on Shanklin and Landwehr data.

Our computational procedure for any station and for any season is, then, as follows:

1. Extract the observed vertical distribution of clouds for the station and season for each eighth of cloud cover from the RAWDAB tapes.

2. Apply the smoothing technique of Eq. (2) to obtain a smooth distribution with height for each eighth of cloud cover.

3. Construct a cumulative distribution for each eighth of cloud cover (such a distribution is illustrated in Table 2).

4. Obtain from Fig. 4 the values of $c(\alpha, k)$ for all desired look angles for each value of $k$, i.e., for each cloud cover amount, in eighths.

5. For each elevation, perform the multiplication and summation indicated by Eq. (5) for each desired look angle.

6. Transform the CFLOS probability as a function of slant range and look angle to a probability as a function of range and look angle by substituting $R=l / \sin \alpha$ for height in the probability function (see Table 3).

Some illustrative results are shown in the three righthand columns of Table 2. Although this procedure sounds complicated, it can be quickly and easily carried out by a set of computer programs. For this study, 
TABLE 3. Height/range conversion table.

\begin{tabular}{|c|c|c|c|c|c|c|c|c|c|}
\hline \multicolumn{2}{|c|}{ Height } & \multicolumn{3}{|c|}{$\begin{array}{c}\text { Slant } \\
\text { range } R \\
(\mathrm{~km}) \\
\text { Look angle } \\
\text { (deg) }\end{array}$} & \multicolumn{2}{|c|}{ Height } & \multicolumn{3}{|c|}{$\begin{array}{c}\text { Slant } \\
\text { range } R \\
(\mathrm{~km}) \\
\text { Look angle } \\
\text { (deg) }\end{array}$} \\
\hline$(\mathrm{ft})$ & $(\mathrm{km})$ & 60 & 45 & 30 & $(\mathrm{ft})$ & $(\mathrm{km})$ & 60 & 45 & 30 \\
\hline 100 & 0.03 & 0.04 & 0.04 & 0.06 & 11,000 & 3.35 & 3.87 & 4.74 & 6.70 \\
\hline 200 & 0.06 & 0.07 & 0.09 & 0.12 & 12,000 & 3.66 & 4.23 & 5.18 & 7.32 \\
\hline 300 & 0.09 & 0.10 & 0.13 & 0.18 & 13,000 & 3.96 & 4.57 & 5.60 & 7.92 \\
\hline 400 & 0.12 & 0.14 & 0.17 & 0.24 & 13,124 & 4.00 & 4.62 & 5.66 & 8.00 \\
\hline 500 & 0.15 & 0.17 & 0.21 & 0.30 & 14,000 & 4.27 & 4.93 & 6.04 & 8.54 \\
\hline 700 & 0.21 & 0.24 & 0.30 & 0.42 & 15,000 & 4.57 & 5.28 & 6.46 & 9.14 \\
\hline 1,000 & 0.31 & 0.36 & 0.44 & 0.62 & 16,000 & 4.88 & 5.64 & 6.90 & 9.76 \\
\hline 1,500 & 0.46 & 0.53 & 0.65 & 0.92 & 16,405 & 5.00 & 5.77 & 7.07 & 10.00 \\
\hline 2,000 & 0.61 & 0.70 & 0.86 & 1.22 & 17,000 & 5.18 & 5.98 & 7.33 & 10.36 \\
\hline 3,000 & 0.91 & 1.05 & 1.29 & 1.82 & 18,000 & 5.49 & 6.34 & 7.76 & 10.98 \\
\hline 3,281 & 1.00 & 1.16 & 1.41 & 2.00 & 19,000 & 5.79 & 6.69 & 8.19 & 11.58 \\
\hline 4,000 & 1.21 & 1.40 & 1.71 & 2.42 & 19,686 & 6.00 & 6.93 & 8.49 & 12.00 \\
\hline 5,000 & 1.52 & 1.76 & 2.15 & 3.04 & 20,000 & 6.10 & 7.04 & 8.63 & 12.20 \\
\hline 6,000 & 1.83 & 2.11 & 2.59 & 3.66 & 22,967 & 7.00 & 8.08 & 9.90 & 14.00 \\
\hline 6,562 & 2.00 & 2.31 & 2.83 & 4.00 & 25,000 & 7.62 & 8.80 & 10.78 & 15.64 \\
\hline 7,000 & 2.13 & 2.46 & 3.01 & 4.26 & 26,248 & 8.00 & 9.24 & 11.31 & 16.00 \\
\hline 8,000 & 2.44 & 2.82 & 3.45 & 4.88 & 29,529 & 9.00 & 10.39 & 12.73 & 18.00 \\
\hline 9,000 & 2.74 & 3.16 & 3.88 & 5.48 & 30,000 & 9.14 & 10.55 & 12.93 & 18.28 \\
\hline 9,843 & 3.00 & 3.46 & 4.24 & 6.00 & 32,808 & 10.00 & 11.54 & 14.14 & 20.00 \\
\hline 10,000 & 3.05 & 3.52 & 4.31 & 6.10 & & & & & \\
\hline
\end{tabular}

we considered only Columbia, during four seasons. Look angles of $30^{\circ}, 45^{\circ}$ and $60^{\circ}$ and 30 elevations (up to $30,000 \mathrm{ft}$ ) were examined.

\section{Error analysis}

The root-mean-square errors of five different methods of estimating CFLOS, together with their biases, were computed by Lund (1966). Lund's criterion for estimating errors was sunshine data-a portion of the same data from which he derived the coefficients for the five methods. Unfortunately, we have no such objective measures against which our computed probabilities can be tested. Therefore, for our error analysis, it was necessary to estimate the magnitude of the errors in the $c$ and $f$ functions and propagate them through the computation indicated in Eq. (1). The error-propagation rules, assuming independence of errors, are given in

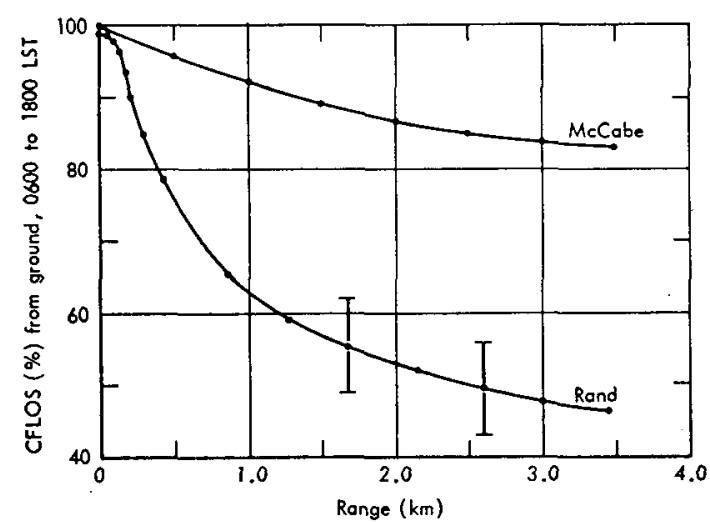

FIG. 6. CFIOS at $45^{\circ}$ look angle over Columbia, Mo. (winter) compared with Quayle's results.
Worthing and Geffner (1943), i.e.,

$$
\begin{gathered}
p^{2}(l, \alpha, k)=f^{2}(l, k) p_{c}{ }^{2}+c^{2}(\alpha, k) p_{f}^{2}, \\
p(l, \alpha)=\left[\sum_{k=0}^{8} p^{2}(l, \alpha, k)\right]^{\frac{k}{2}},
\end{gathered}
$$

where $p_{c}$ is the probable error in $c, p_{f}$ the probable error in $f$, and $p(l, \alpha)$ the probable error in the CFLOS estimate.

We estimated $p_{c}$ by first comparing the smoothed curves of Fig. 4 with the raw data of Shanklin and Landwehr (1971) for a look angle of $45^{\circ}$. The discrepancies ranged from $-1.0 \%$ to $+0.3 \%$. Comparing the smoothing done on the same data by two analysts, we found that the maximum difference was $1.0 \%$. For the purposes of this demonstration, therefore, we assume a probable error of $1.0 \%$ as a safe estimate for the function $c(\alpha, k)$.

The probable errors of the function $f(l, k)$ are a bit harder to derive. The standard errors in estimates of the probability of occurrence of a cloud amount for the raw data range from less than $1.0 \%$ (for the more frequent occurrences) to $5.0 \%$ (for the less frequent occurrences). The effect of the smoothing is difficult to estimate, but for purposes of crudely estimating the reliability, we will assume that $p_{f}$ (the probable error of the function $f$ ) is $3.0 \%$.

Using the above estimates of the probable errors and using values of $c$ taken from Fig. 4 for a look angle of $45^{\circ}$, and values of $f$ taken from Table 2 for cloud heights of 2000, 4000 and $6000 \mathrm{ft}$, we evaluated Eqs. (6) and (7). In all cases, the first term on the right-hand side of Eq. (6) was so much less than the second term that 

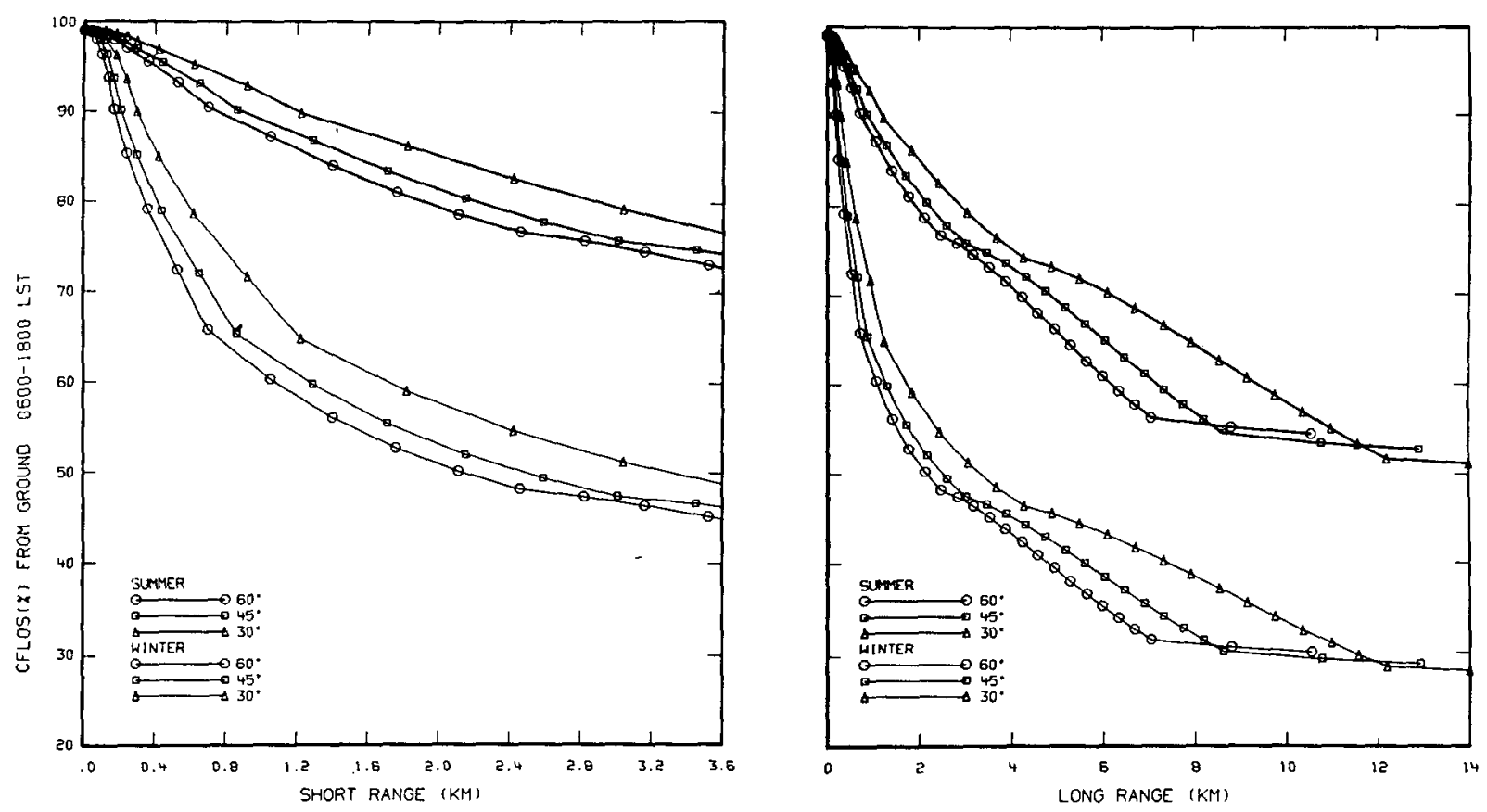

FIG. 7. CFLOS probabilities, summer and winter, at Columbia, Mo.

the variation with altitude was undetectable, because $c$ and $p_{f}$ are assumed to be invariant with height. The resultant probable error at a $45^{\circ}$ look angle was found to be $\pm 6.5 \%$, a value that is consonant with the results of Lund. These errors are plotted in Fig. 6 for comparison with the results based on the data of Quayle $e t$ al. This comparison strongly suggests that those estimates of CFLOS probability are entirely too high.

\section{Seasonal CFLOS probabilities at Columbia}

An example of the patterns of CFLOS probabilities, looking upward from the ground during the hours 0600-1800 LST (local standard time) and using the techniques just discussed, is shown for Columbia $\left(38^{\circ} 58^{\prime} \mathrm{N}-92^{\circ} 22^{\prime} \mathrm{W}\right)$ in Figs. 7 and 8 . The seasons are paired so that summer (June-August) and winter (December-February) can be compared on one graph, and spring (March-May) and fall (September-November) on another. The curves indicate the probability of CFLOS for three viewing angles at short ranges (left) and long ranges (right).

Columbia (and much of the eastern United States) is dominated by unstable tropical maritime ( $\mathrm{mT}$ ) air in summer, and modified, but stable, polar continental (cP) air in winter. Winter data from ETAC from 1945-68 show that Columbia is clear on $\sim 8 \%$ of the days and has scattered $(<5 / 8)$ clouds on only $10 \%$. The remaining broken-to-overcast cloud cover results from modification along the flow or by interaction of $\mathrm{cP}$ and moist $\mathrm{m} T$ along frontal lines. Resulting low stratus and stratocumulus-type clouds dominate and give the rapid dip of the winter curves (Fig. 7) at the short ranges. The "bump" in the curves between 4 and 6 $\mathrm{km}$ indicate the presence of middle clouds, largely from frontal overrunning during this season.

Summer weather is less complicated by extensive storms, although line squalls and thunderstorms with multiple layers account for much of the erratic slope in these curves (Fig. 7). The predominant clouds are cumulus, within the dominating moist, unstable $\mathrm{mT}$ air mass. They form at a higher level than the winter stratus and thereby allow a more gradual decrease in the CFLOS.

The fall and spring curves (Fig. 8) show similar characteristics. Since there is a rapid decrease in the CFLOS probability at the short ranges, and a marked "bump" at middle ranges, these curves indicate a predominant winter influence.

\section{Concluding remarks}

The use of station data for determining eighths of cloud cover at given heights, and the use of CFLOS measurements for known clouds, provide greater flexibility for determining local CFLOS probabilities around the world at given ranges. These CFLOS data are clearly more sensitive to the climate zone of the stations of interest than are earlier data.

As is stated at the beginning of this paper, we reviewed the entire computational procedure for estimating CFLOS probabilities originally developed by McCabe (1965) because estimates based on that work in Quayle et al. (1968) seemed unreasonably optimistic. The CFLOS probabilities for a $45^{\circ}$ look angle estimated by Quayle et al., for Columbia in winter, were compared 

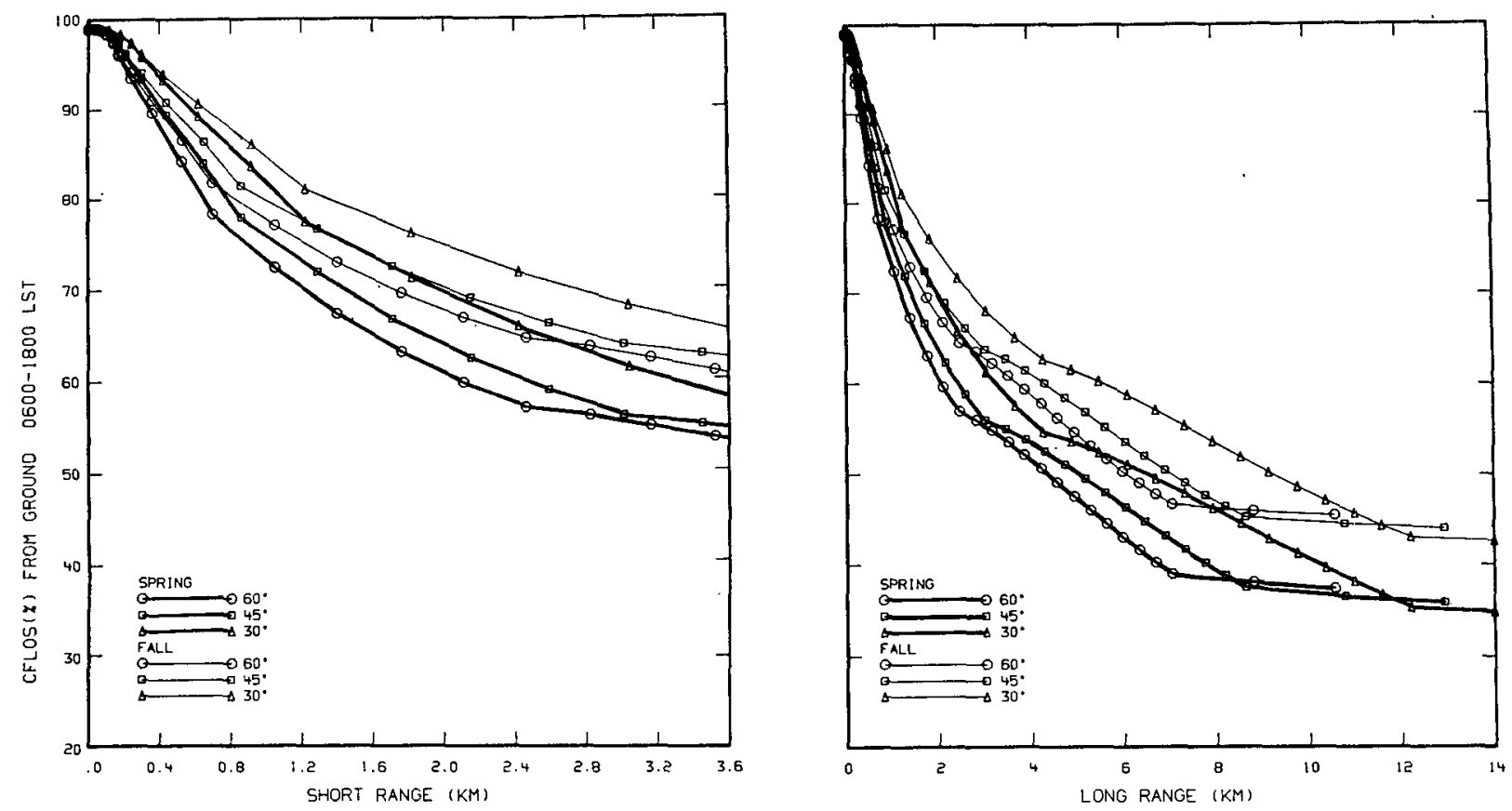

Fig. 8. CFLOS probabilities, spring and fall, at Columbia, Mo.

with our results in Fig. 6 . We have carefully calculated this smooth curve and believe that the detail we have captured at the short ranges is realistic. We believe it properly reflects the asymptotic behavior at longer ranges and that the differences in probabilities between, say, the 50 and the $85 \%$ shown here for $2.6 \mathrm{~km}$ range, excludes any credence that the Quayle curve truly represents the probability of a CFLOS.

From the information currently available, it appears that Shanklin and Landwehr's measurements in Fig. 4 for $c(\alpha, k)$ are probably the best data presently available. These are measurements of actual cloud conditions, whereas McCabe's data represent estimates of the difference between ground observations and what might be seen from above. Refinement of Fig. 4 will require a continued program of direct measurement at Columbia and elsewhere to establish a good climatological base, especially as related to the effects of different cloud genera on CFLOS.

Measurements made without reference to surface observation, such as those of Bertoni, provide some insight into the CFLOS problem and have been helpful, but they do not provide a realistic means for utilizing available climatological data.

Findings from direct measurement programs such as Appleman's and that of Shanklin and Landwehr might constitute the final steps for establishing definitive CFLOS probabilities. Huschke (1971) outlines a scheme for relating these data to air masses of similar origins and to the general circulation patterns of both the Northern and Southern Hemispheres. Therefore, data collected according to this scheme at carefully chosen test sites across the United States could form the basis for relationships between ground observations and CFLOS that would be climatologically reliable for worldwide application, especially in areas where data are sparse.

Acknowledgments. The authors wish to express their sincere appreciation to those in the Environmental Technical Applications Center of the Air Force for their cooperation in making available the latest magnetic tapes of daily synoptic weather reports, and to Iver A. Lund of the Air Force Cambridge Research Laboratories for his review and helpful comments.

Special thanks are also extended to several Rand colleagues for their invaluable assistance: R. E. Huschke assisted in interpreting data from programs designed to interrogate the Rand Weather Data Bank, Marsha Dade assisted with the mathematical calculations, and Rose Heirschfeldt did the programming necessary to obtain graphs from the FR-80 Integrated Graphics System.

\section{REFERENCES}

Appleman, H. S., 1962: A comparison of simultaneous aircraft and surface cloud observations. $J$. Appl. Meteor., 1, 548-551.

Bertoni, E. A., 1967: Clear lines of sight from aircraft. Air Force Surveys in Geophysics, No. 196, $181 \mathrm{pp}$.

DeBary, E., and F. Möller, 1963: The vertical distribution of clouds. J. A ppl. Meteor., 2, 806-808.

Greaves, J. R., D. B. Spiegler and J. H. Willand, 1971 : Development of a global cloud model for simulating earth-viewing space missions. Final Rept., Contract NAS 8-25812, Allied Research Associates, Inc. 
Huschke, R. E., 1971: Use of weather-information in determining cost/performance and force-mix tradeoffs: Weather and warplanes I. Rept. R-740-PR, Santa Monica, The Rand Corporation, $41 \mathrm{pp}$.

Lund, I. A., 1965: Estimating the probability of clear lines-ofsight from sunshine and cloud cover observations. $J . A p p l$. Meteor., 4, 714-722.

$\longrightarrow, 1966$ : Methods for estimating the probability of clear linesof-sight, or sunshine, through the atmosphere. $J$. Appl. Meteor., 5, 625-630.

- , and M. D. Shanklin, 1972: Photogrammetrically determined cloud-free lines-of-sight through the atmosphere. $J . A p p l$. Meteor., 11, 773-782.
McCabe, J. T., 1965: Estimating mean cloud and climatological probability of cloud-free line-of-sight. Tech. Rept. 186, Air Weather Service (MATS), $26 \mathrm{pp}$.

Quayle, R. G., J. M. Meserve and H. L. Crutcher, 1968: Probability of penetrable optical path for high intensity, high contrast, optical targets. Vol. IV-North America. National Weather Records Center, $18 \mathrm{pp}$.

Shanklin, M. D., and J. B. Landwehr, 1971 : Photogrammetrically determined cloud-free lines-of-sight at Columbia, Missouri. Final Rept., AFCRL-71-0273, Air Force Cambridge Research Laboratories, $185 \mathrm{pp}$.

Worthing, A. G., and Joseph Geffner, 1943: Treatment of Experimental Data. New York, Wiley, 344 pp. 